Research Article

\title{
The Role of Overexpressed Apolipoprotein AV in Insulin-Resistant Hepatocytes
}

\author{
Wang Zhao, ${ }^{1}$ Yaqiong Liu, ${ }^{1}$ Xiaobo Liao, ${ }^{2}$ and Shuiping Zhao $\mathbb{D}^{1}$ \\ ${ }^{1}$ Department of Cardiovascular Medicine, The Second Xiangya Hospital, Central South University, No. 139 Middle Renmin Road, \\ Changsha, Hunan 410011, China \\ ${ }^{2}$ Department of Cardiovascular Surgery, The Second Xiangya Hospital, Central South University, No. 139 Middle Renmin Road, \\ Changsha, Hunan 410011, China \\ Correspondence should be addressed to Shuiping Zhao; zhaosp@csu.edu.cn
}

Received 31 January 2019; Revised 16 July 2019; Accepted 16 August 2019; Published 21 April 2020

Academic Editor: Kui Li

Copyright (๑) 2020 Wang Zhao et al. This is an open access article distributed under the Creative Commons Attribution License, which permits unrestricted use, distribution, and reproduction in any medium, provided the original work is properly cited.

\begin{abstract}
In this paper, we sought to explore the relationship between apolipoprotein $\mathrm{AV}(A P O A V)$ overexpression and insulin resistance in hepatocytes. The insulin-resistant HepG2 cell model was constructed, and then, $A P O A V$-overexpressed HepG2 cells (B-M) were induced by infecting with a recombinant adenovirus vector. Microarray data were developed from B-M samples compared with negative controls (A-con), and the microarray data were analyzed by bioinformatic methods. $A P O A V$-overexpression induced 313 upregulated genes and 563 downregulated ones in B-M sample. The differentially expressed genes (DEGs) were significantly classified in fat digestion and absorption pathway. Protein-protein interaction network was constructed, and AGTR1 (angiotensin II receptor type 1) and P2RY2 (purinergic receptor P2Y, G-protein coupled 2) were found to be the significant nodes closely related with G-protein related signaling. Additionally, overexpression of $A P O A V$ could change the expression of Glut 4 and release the insulin resistance of hepatic cells. Thus, $A P O A V$ overexpression may prevent the insulin resistance in liver cells by mediating the genes such as AGTR1 and P2RY2.
\end{abstract}

\section{Introduction}

Insulin resistance has become an increasingly common metabolic syndrome in people around the word. The main understanding of the mechanism for insulin resistance is the glycometabolism disorder in liver [1]. Insulin resistance results in excessive glucose consumption in liver, which contributes to the development of hyperglycemia [2]. In addition, hyperglycemia is frequently associated with metabolic syndromes such as hypertension, coronary heart disease, and diabetes [3,4]. Type 2 diabetes (T2D) is a common metabolic disorder that characterizes high blood sugar, insulin deficiency, and insulin resistance. T2D (noninsulin-dependent) is the risk for cardiovascular disease that is the main cause for death of diabetes patients [5].

Recently, apolipoprotein AV (APOAV), also served as the APOA5, has been found to be a novel apolipoprotein and to play a key role in the processes of insulin resistance and lipid metabolism $[6,7]$. APOAV is mainly expressed in liver and closely associated with high-density lipoprotein [8]. Moreover, APOAV is also found to participate in the metabolism of triglycerides by stimulating hydrolysis of triglycerides, reducing the lipoprotein production rate, and facilitating clearance of triglyceride-rich lipoprotein [9]. A previous study suggests that $A P O A V$ is downregulated in insulin-treated mice and the expression of $A P O A V$ a contributor for hypertriglyceridemia [10]. The decreased expression $A P O A V$ is involved in insulin resistance-related hypertriglyceridemia [6]. APOAV seems to be closely related with insulin resistance in diabetics, but whether the overexpression of $A P O A V$ could prevent insulin resistance needs to be further determined.

Microarray technology has been widely used to generate the gene expression data on a genomic scale [11]. In this paper, we performed the microarray analysis for the human hepatoma (HepG2) cells to discover the differentially 
expressed genes (DEGs) induced by APOAV overexpression. HepG2 cells, which are characterized by the common physiological function in glucose metabolism with normal hepatic cells [12], were used in this study. Therefore, in the present study, we sought to explore the potential mechanism of relationship between $A P O A V$ expression and insulin resistance in hepatocytes.

\section{Materials and Methods}

2.1. Insulin-Resistant HepG2 Cell Model Construction. HepG2 cell lines (Cell Culture Center of Peking Union Medical Science, Beijing, China) were cultured at $37^{\circ} \mathrm{C}$ in Dulbecco's modified eagle medium (DMEM) supplemented with $10 \%(\mathrm{v} / \mathrm{v})$ FBS (fetal bovine serum) and $100 \mathrm{IU}$ penicillin/streptomycin/amphotericin in a humidified $5 \% \mathrm{CO}_{2}$ atmosphere. When entering the logarithmic phase, the cells were washed by PBS, and then digested with trypsin enzyme. After the cells turned round, cell suspension was put into $15 \mathrm{ml}$ centrifuge tube and centrifuged at $1000 \mathrm{rpm}$ for $3 \mathrm{~min}$ and inoculated in 96 -well plates at the density of $5 \times 10^{3}$ cells/ $\mathrm{cm}^{2}$.

In order obtain the insulin-resistant HepG2 cell model, the cells were treated with insulin at a series of concentration gradients. The insulin-resistant HepG2 cell model was induced as described previously [13]. Briefly, HepG2 cells were subjected to $24 \mathrm{~h}$-culture of $5 \times 10^{-5}, 5 \times 10^{-6}, 5 \times 10^{-7}$, and $5 \times 10^{-8} \mathrm{~mol} / \mathrm{L}$ bovine insulin, followed by incubating in $10^{-9} \mathrm{~mol} / \mathrm{L}$ insulin for $24 \mathrm{~h}$. And to determine whether the insulin model was successfully constructed, glucose uptake rate and liver glycogen synthesis were measured. For glucose uptake rate measurement, the supernatant was absorbed into a $15 \mathrm{~mL}$ centrifuge tube at $1500 \mathrm{rpm} / 6 \mathrm{~min}$, and transferred to a $1.5 \mathrm{ml} \mathrm{EP}$ tube. After the supernatant was diluted 6 times with distilled water, and the glucose uptake rate was determined by the urine glucose assay kit-oxidase assay according to the manufacturer's instructions. Glucose uptake rate $=$ (preinduced glucose concentration-postinduced glucose concentration)/preinduced glucose concentration. For glycogen synthesis measurement, (1) the cells were digested with trypsin, centrifuged (3000 g, $5 \mathrm{~min}$ ) and weighed; (2) hydrolysis: sample weight (mg): lye volume $(\mu \mathrm{L})=1: 3$, boiled in boiling water for $20 \mathrm{~min}$; (3) liver glycogen detection solution (1\%); (4) the standard glucose solution: the standard glucose solution was prepared into 25 , $12.5,6.25,3.125$, and $1.5625 \mathrm{~g} / \mathrm{ml}$ with distilled water; (5) OD620 nm value of each tube was measured; and (6) standard curve was drawn for calculating the liver glycogen concentration.

2.2. Western Blot Analysis. To determine the native APOAV expression in the induced model cells and insulin-resistant HepG2 cells infected by recombinant adenovirus, western blot analysis was performed. Briefly, the cells were lysed in a radioimmunoprecipitation assay (RIPA) buffer supplemented with for proteins extraction, and total protein was quantified with the bicinchoninic acid (BCA) assay. Total proteins $(30 \mu \mathrm{g} /$ lane $)$ were separated via $12 \%$ SDS-PAGE and then transferred to a polyvinylidene difluoride (PVDF) membrane. After blocked with 5\% skimmed milk for $1 \mathrm{~h}$, the membrane was incubated with primary antibodies (APOAV5, 1:1000; beta actin, $1: 1000$ ) at $4^{\circ} \mathrm{C}$ overnight. Following washing with TBST for 3 times, the membrane was incubated with secondary antibody (antirabbit IgGHRP, $1: 5000$; antimouse IgG-HRP, $1: 5000$ ) for $2 \mathrm{~h}$ at room temperature. Finally, the proteins were visualized using the ECL chemiluminescence reagent.

2.3. Recombinant Plasmid Vector with APOAV. The APOAV gene was amplified by PCR with the primers, such as $5^{\prime}$-ACA CGGATCCATGGCAAGCATGGCTGCCGT-3' (forward) and $5^{\prime}$-ACACGAATTCTCAGGGGTCCCCCAGATG-3' (reverse). After the plasmid pHBAd-MCMV-GFP was digested with BamH1 and EcoR1, the recombinant plasmid vector was constructed by connecting with $A P O A V$ at $4^{\circ} \mathrm{C}$ overnight. The positive recombinant clone was named as pHBAd-MCMV-APOAV-GFP. The expressions of APOAV and GFP were regulated by cytomegalovirus (CMV) promoter.

2.4. Insulin-Resistant HepG2 Cells Infection by Recombinant Adenovirus. HEK293 was the common cell for packing the recombinant adenoviruses because of its E1 genes. To generate recombinant adenovirus vectors with $A P O A V$ expression, the pHBAd-MCMV-APOAV-GFP and adenovirus skeleton plasmid vector of $\mathrm{pHBAd}-\mathrm{BHG}$ were coinfected into HEK293 cells with the lipofectamine 2000 kit following the manufacturer's instruction. After $6 \mathrm{~h}$ transformation, the recombinant adenoviruses were harvested. Then, the insulin-resistant HepG2 cells were cultured and infected with the recombinant adenovirus vector for $48 \mathrm{~h}$. The successful infection by recombinant adenovirus was determined, when the expression of GFP was observed by fluorescence microscopy.

2.5. RNA Isolation. Total RNA of insulin-resistant HepG2 cells with APOAV overexpression (B-M) and negative controls (A-con) was isolated using TRIzol reagent (Invitrogen, Carlsbad, CA) according to the manufacturer's protocol. The expected quality of RNA was determined by measuring the absorbance ratios (A260/A280) between 1.8 and 2.0 under the spectrophotometer.

2.6. Microarray Analysis. The RNA library was constructed by using the NEBNext ultra directional RNA library prep kit for illumina (New England Biolabs, Ipswich, MA, USA) following the manufacturer's instruction. After the library quality was assessed by Aglient 2100 Bioanalyzer (Agilent Technologies, Palo Alto, CA), the next-generation sequencing was performed based on the Illumina HiSeq4000 platform (Illumina Inc., San Diego, CA). Read counts were normalized by TMM (trimmed mean of $M$ values) in the edgeR package [14]. Then, the read count data were transformed to $\log 2$-counts per million $(\log C P M)$ for gene expression by the limma-voom package [15]. The differential 
gene expression analysis between B-M and A-con cells was carried out by the limma package in $R[16,17]$. Genes with $P<0.05$ and $\log _{2} \mid$ fold change $\mid \geq 1$ were considered to be significantly different. Cluster analysis for DEGs was performed by gplots in $R$ [18].

2.7. Function Analysis. The functionally associated genes were classified with the aim to explore the altered function and pathways in B-M cells. The GO (Gene Ontology) functions and KEGG (Kyoto Encyclopedia of Genes and Genomes) pathways were analyzed following the protocol of DAVID (version: 6.8) [19]. The count $\geq 2$ and $P$ value $<0.05$ were set as the cutoff value.

2.8. Protein-Protein Interaction Network Analysis. STRING (Search Tool for the Retrieval of Interacting Genes/Proteins) database is a collection of protein interaction pairs, including physical and functional interactions [20]. The gene interactions analysis was performed based on protein interactions by STRING version: 10.0 [21]. The protein-protein interaction (PPI) network was constructed by protein interactions with PPI score $\geq 0.4$ and visualized by Cytoscape (version: 3.2.1) [22]. Then, the node degrees were calculated for screening the hub nodes.

2.9. Module Analysis. Cytoscape plugin ClusterONE [23] provided network cluster analysis for screening significant modules. Modules with $P<1.0 E-4$ were considered to be significant. The GO functions and KEGG pathways significantly enriched by module genes were further analyzed by DAVID (version: 6.8) software. Subsequently, the module genes with high node degrees were screened out as feature genes.

2.10. Disease-Associated Gene Analysis. The Comparative Toxicogenomics Database (CTD, http://ctdbase.org/) [24] provides the gene-disease relationships recorded in the previous studies. All of the marker genes associated with T2D were retrieved from the CTD database.

\section{Verification Experiments}

3.1. AML12 Cells with APOAV Overexpression. Mouse liver cells of AML12, purchased from the Chinese Academy of Sciences, Shanghai, China, were divided into the blank group, normal control group (empty vector), and APOAV group (APOAV overexpression). The recombinant plasmid vectors with $A P O A V$ overexpression were constructed according to the method mentioned above. Then, the recombinant plasmid vectors were transfected into AML12 cells. The AML12 cells successfully infected with the recombinant adenovirus vector were collected under fluorescence microscopy.

3.2. RT-qPCR. Total RNA from AML12 cell lines was extracted according to the method mentioned above, and the expression level of $A P O A V$ was determined according to the
qRT-PCR with the following amplification conditions: predegeneration at $95^{\circ} \mathrm{C}$ for $3 \mathrm{~min}$, followed by 40 cycles at $95^{\circ} \mathrm{C}$ for $30 \mathrm{sec}, 30 \mathrm{sec}$ at $60^{\circ} \mathrm{C}$. GAPDH was used as the internal control, and the primer sequences were as follows: APOAV: forward, 5'-TCCTCGCAGTGTTCGCAAG-3', and reverse, 5' - GAAGCTGCCTTTCAGGTTCTC-3'; GAP DH: forward, $5^{\prime}$-GGTGAAGGTCGGTGTGAACG-3', and reverse, 5'-CTCGCTCCTGGAAGATGGTG-3'. All reactions were performed in triplicate, and relative expressions of $A P O A V$ in cell lines were calculated by the $2^{-\Delta \Delta c t}$ method.

3.3. Enzyme-Linked Immunosorbent Assay (ELISA). After infected with recombinant adenovirus vectors, the cells were adjusted to $5 \times 10^{4}$ cells $/ \mathrm{cm}^{2}$ and then maintained at $37^{\circ} \mathrm{C}$ overnight. When the cells grew up to $50 \%$, the cells were treated with $5 \times 10^{-7} \mathrm{~mol} / \mathrm{L}$ insulin for $48 \mathrm{~h}$. The level of glucose was evaluated by the ELISA kit (Beijing Pulilai gene co. LTD, Beijing, China) following the manufacturer's protocol.

Furthermore, the levels of Glut 4 in the three groups were measured with the ELISA kit (Shanghai Enzyme-linked Biotechnology Co., Ltd, Shanghai, China) according to the manufacturer's protocol.

3.4. Statistical Analysis. All the data were expressed as mean \pm SD (standard deviation). Statistical analyses were performed with Graphpad prism 5 (Graphpad Software, San Diego, CA). The comparison among groups was performed by one-way ANOVA (one-way analysis of variance). Differences between groups were compared by LSD (least significant difference test) method. $P<0.05$ was considered significant.

\section{Results}

4.1. The Construction of Insulin-Resistant HepG2 Cell Model. As illustrated in Figures 1(a) and 1(b), compared with normal control, the insulin groups with $5 \times 10^{-8}, 5 \times 10^{-7}$, and $5 \times 10^{-8}$ could significantly decrease the glucose uptake rate and liver glycogen synthesis of HepG2 cells. Specially, the insulin concentration of $5 \times 10^{-7}$ was the most significant effect, which was selected for the following experiment. Furthermore, the glucose uptake rate and liver glycogen synthesis were used as the criteria for insulin-resistant cell model construction. Moreover, the results of western blot analysis showed that APOAV 5 levels in insulin-resistant HepG2 cells were decreased in comparison to normal controls (Figure 1(c)).

4.2. Insulin-Resistant HepG2 Cells Infected with Recombinant Adenoviral Vectors. HEK239 were independently infected with APOAV overexpressed recombinant adenoviruses and GFP control. As shown in Figure 2(a), the cells changed to round and plaques appeared obviously, which illustrated that the recombinant vectors were constructed successfully. After adenovirus vectors transfection, the GFP-positive cells were detected with the green 


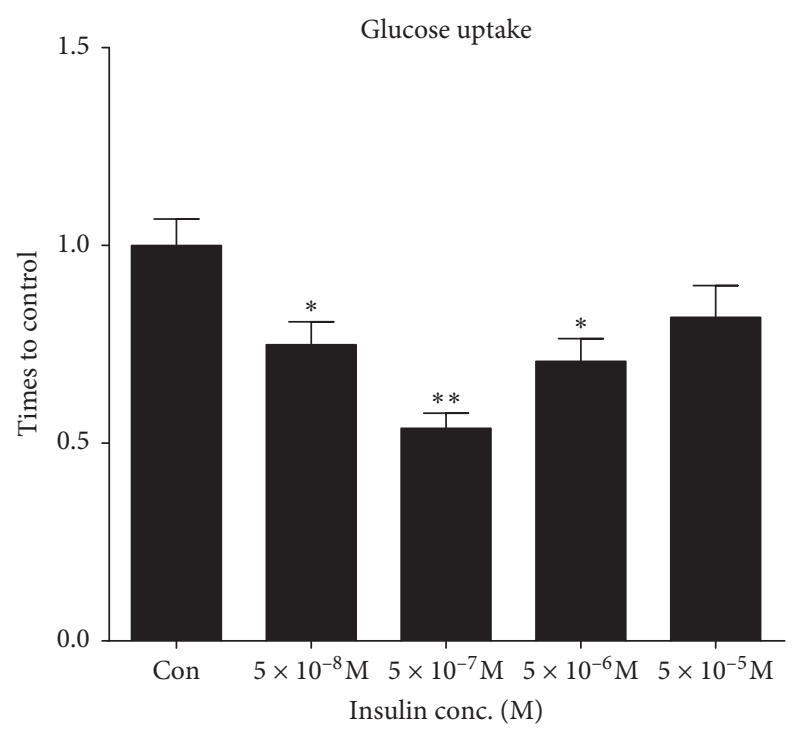

(a)

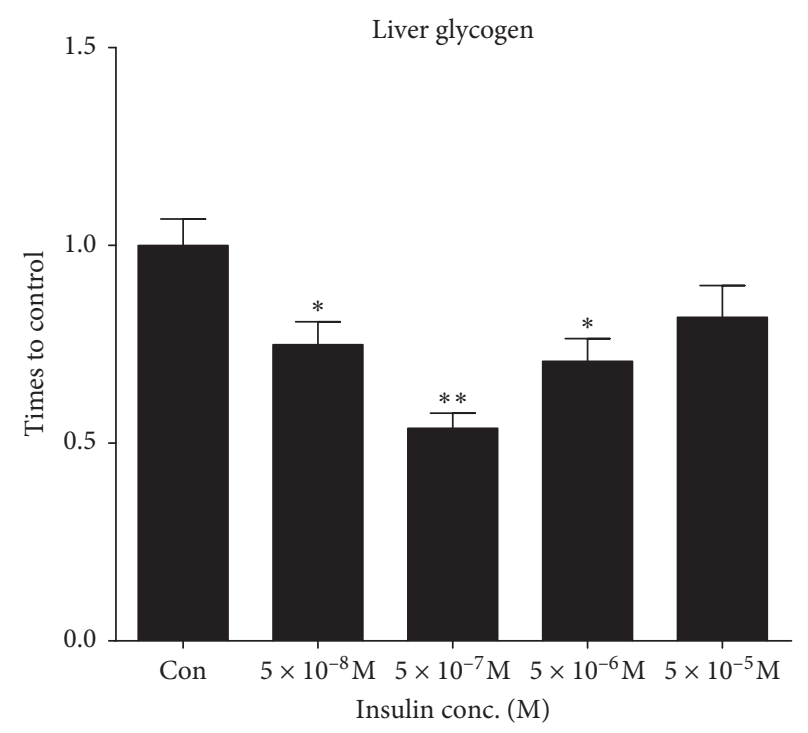

(b)

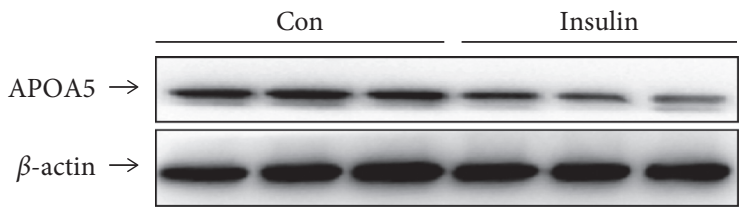

(c)

FIGURE 1: Insulin-resistant HepG2 cell model construction. (a) Glucose uptake rate and (b) liver glycogen synthesis after HepG2 cells treated with $5 \times 10^{-5}, 5 \times 10^{-6}, 5 \times 10^{-7}$, and $5 \times 10^{-8} \mathrm{~mol} / \mathrm{L}$ bovine insulin. (c) APOAV protein expression in insulin-resistant HepG2 cells.

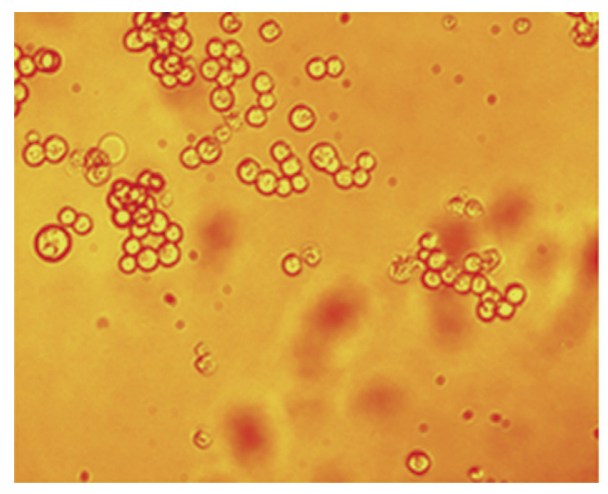

B-M

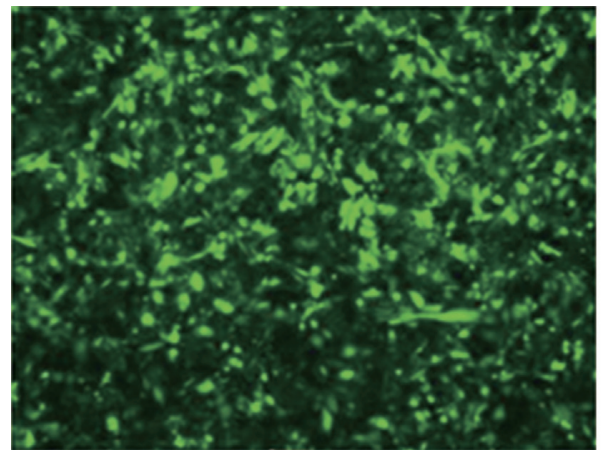

B-M

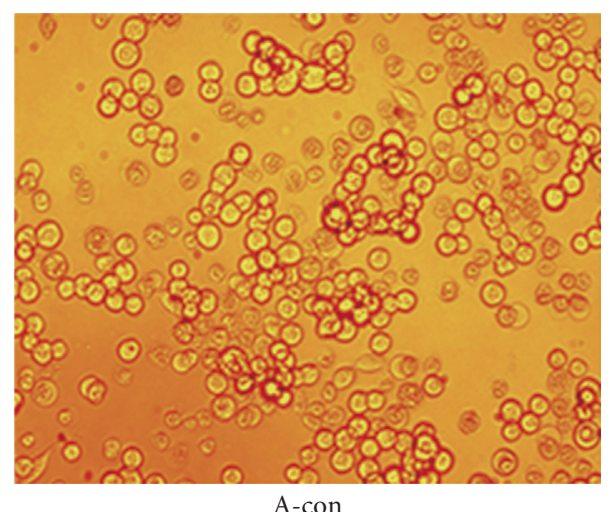

(a)

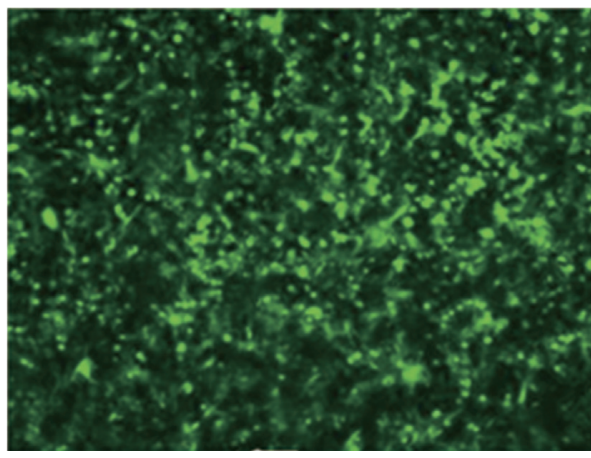

A-con

(b)

Figure 2: Continued. 


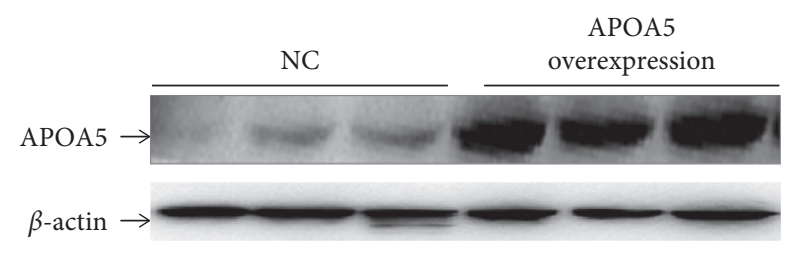

(c)

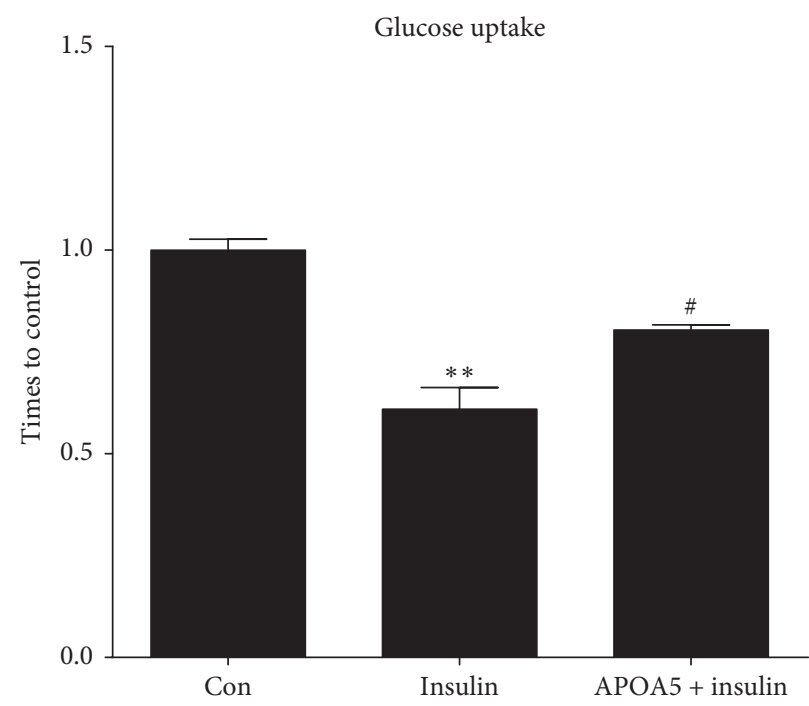

(d)

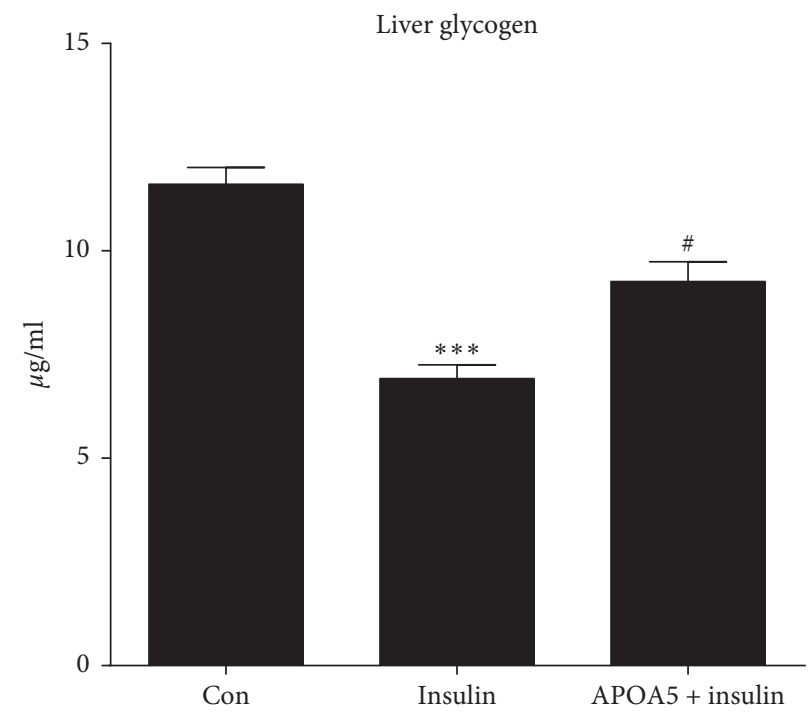

(e)

FIGURE 2: Successful construction of the insulin-resistant HepG2 cells with APOAV overexpression. (a) Recombinant adenoviruses packing HEK293 cells were infected with recombinant adenoviruses with APOAV overexpression. (b) The fluorescence images of insulin-resistant HepG2 cells with APOAV overexpression. B-M: insulin-resistant HepG2 cells with APOAV overexpression; A-con: negative controls. (c) Western blot analysis for APOAV 5 expression in control and insulin-resistant HepG2 cells with APOAV overexpression. (d) Glucose uptake rate and (e) liver glycogen synthesis in normal control, insulin-resistant HepG2 cells, and insulin-resistant HepG2 cells with APOAV overexpression.

fluorescence (Figure 2(b)). Moreover, compared with the controls, the expression of APOAV 5 protein in insulinresistant HepG2 cells infected by recombinant adenovirus was upregulated (Figure 2(c)). In comparison to the insulin-resistant HepG2 cells, the glucose uptake rate and liver glycogen synthesis in cells infected by recombinant adenovirus were significantly increased (both, $P<0.05$; Figures 2(d) and 2(e)), respectively. All these suggested that insulin-resistant HepG2 cells were successfully infected by recombinant adenovirus. 
4.3. Identification of DEGs. The gene expression difference between B-M and A-con cells was assessed by microarray array analysis. The comparison of the gene expression pattern for B-M and A-con cells showed 876 genes differentially expressed $\left(P<0.05\right.$ and $\log _{2} \mid$ fold change $\left.\mid \geq 1\right)$, of which 313 were upregulated and 563 were downregulated. The hierarchical clustering were performed in two dimensions such as samples and genes (Figure 3), suggesting the clear differentiation was observed between B-M and A-con samples.

4.4. Characterization of Altered Function and Pathways. In order to analyze the potential biological function that was interfered by genes differentially expressed, the DEGs were annotated based on GO and KEGG databases. Results showed that the significantly different genes were classified in different GO categories (BP: biological process, CC: cellar component, and MF: molecular function) and pathways. The upregulated genes were closely related with intrinsic apoptotic signaling pathway by p53 class mediator-related BP, synaptic vesicle membrane-related CC, and calcium-dependent phospholipid binding-related MF. The downregulated genes were enriched in cell-cell signaling-related $\mathrm{BP}$, extracellular region-related CC, and lipid transporter activity-related MF. In addition, the cancer-associated pathways were significantly associated with upregulated genes, such as small cell lung cancer and prostate cancer. The downregulated genes showed close association with systemic lupus erythematosus, $\mathrm{ABC}$ transporters, and legionellosis pathways (Table 1). And all these data reveal the potential processes and pathways that DEGs might be involved.

4.5. PPI Network and Hub Nodes Construction. Total 547 protein interaction pairs with PPI score $\geq 0.4$ were identified in the PPI network. As shown in Figure 4, the PPI network was constructed, which was comprised of 317 nodes and 547 edges. The hub nodes with degree $\geq 10$ in network were screened out (Table 2), such as $\mathrm{H} 2 \mathrm{AFX}$ ( $\mathrm{H} 2 \mathrm{~A}$ histone family member $X$, degree $=19$ ), HDAC9 (histone deacetylase 9, degree =18), ESR1 (estrogen receptor 1, degree =18), BMP2 (bone morphogenetic protein 2 , degree $=17$ ), and CDC6 (cell division cycle 6 , degree $=15$ ), which could be selected as the key genes.

4.6. Significant Modules in PPI Network. With the application of ClusterONE software, two modules were found to be significant. As shown in Figure 5(a), there were 9 nodes in module 1 and 13 nodes in module 2. Most of the module genes showed high node degrees in PPI network (Table 3). In addition, hierarchical clustering revealed the existence of 2 group samples (B-M and A-Con cells) were separated clearly by module genes (Figure 5(b)). All these results determined that the DEGs in modules were the feature genes for B-M and A-Con cells.

DAVID tool summarized the biological importance of the group of functionally related genes with an enrichment score. Function classification for genes in module 1 revealed a series of items, such as alteration of phospholipase C-activating G-protein coupled receptor signaling pathway (GO:0007200), integral component of plasma membrane (GO:0005887), G-protein-coupled purinergic nucleotide receptor activity (GO:0045028), and neuroactive ligandreceptor interaction pathway (hsa04080). Similarly, the module 2 genes were sorted into function classifications, including the nucleosome assembly (GO:0006334), nucleosome (GO:0000786), protein heterodimerization activity (GO:0046982), and systemic lupus erythematosus (hsa05322) pathway (Table 4).

\subsection{Verification Experiments}

4.7.1. APOAV Expression Promoted the Glucose Absorption and Glut4 Levels of AML12 Cell. As shown in Figure 6(a), compared with NC group, the $A P O A V$ expression was significantly upregulated $(P<0.01)$, suggesting the transfection efficiency of $A P O A V$ overexpression lentivirus was satisfactory. After $6 \mathrm{~h}$ of starvation, the glucose consumption of each group was measured by ELISA. The results showed that glucose consumption in group with APOAV overexpression was significantly lower than that in the NC group $(P<0.05$; Figure 6(b)). Additionally, according to Figure 6(c), Glut4 protein level in $A P O A V$ overexpression group was observably increased in comparison to that in $\mathrm{NC}$ group with the empty vector $(P<0.01)$, which indicated that the overexpression of $A P O A V$ could release the insulin resistance of AML12 cells.

\section{Discussion}

In the present study, we performed the microarray profiling of insulin-resistant HepG2 cells with APOAV overexpression to investigate the differential gene expression pattern induced by APOAV overexpression in comparison to the negative controls. Previous evidence shows that insulin resistance is not only the important pathogenesis of T2D but also the root cause of various complications [25]. APOAV is a component of several lipoprotein fractions and has been reported to be negatively correlated with insulin resistance [26]. However, there have been no studies concerning the expression level of genes induced by APOAV overexpression in insulin-resistant hepatocytes. Consequently, we focused on the differential expression profiling relevant to APOAV overexpression in insulin-resistant liver cells.

5.1. Insulin-Resistant Cell Model Construction with HepG2 Cell. Because of the limitation of human trials, most of the fundamental research relevant to insulin resistance has applied cellular models of animals $[27,28]$. HepG2 is derived from human liver embryoma cells, which show many biological properties of hepatocellular [29]. Thereby, HepG2 cell line was reliable for our research. In this paper, the insulin-resistant HepG2 cell model was firstly constructed and infected with APOAV overexpressed recombinant adenoviruses. The green fluorescence imaging illustrated that 


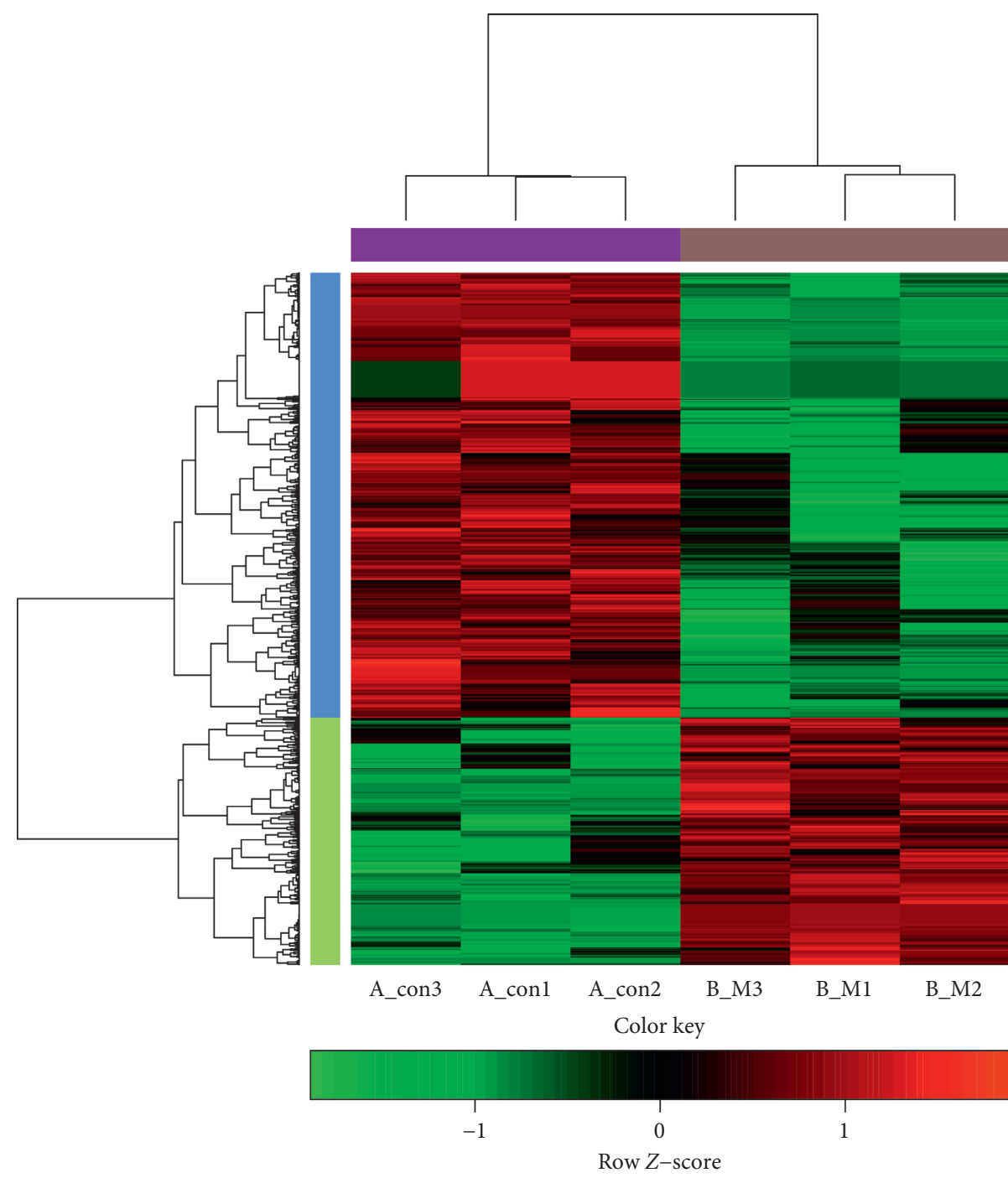

Figure 3: Hierarchical clustering analysis for differentially expressed genes. The color scale at the top illustrates the relative expression level of an mRNA. Red represents a high relative expression and blue represents a low relative expression.

TABLE 1: The biological function and pathways significantly related with differentially expressed genes.

\begin{tabular}{|c|c|c|c|c|}
\hline & Category & Term & Count & $P$ value \\
\hline \multirow{12}{*}{ Up } & \multirow{3}{*}{$\mathrm{BP}$} & $\begin{array}{c}\text { GO:0072332 intrinsic apoptotic signaling pathway } \\
\text { by p } 53 \text { class mediator }\end{array}$ & 4 & $3.37 E-03$ \\
\hline & & GO:0008283 cell proliferation & 10 & $1.77 E-02$ \\
\hline & & GO:0000278 mitotic cell cycle & 11 & $1.85 E-02$ \\
\hline & \multirow{3}{*}{$\mathrm{CC}$} & GO:0030672 synaptic vesicle membrane & 5 & $3.26 E-03$ \\
\hline & & GO:0008021 synaptic vesicle & 6 & $3.65 E-03$ \\
\hline & & GO:0031225 anchored component of membrane & 6 & $7.74 E-03$ \\
\hline & \multirow{3}{*}{ MF } & $\begin{array}{c}\text { GO:0005544 calcium-dependent phospholipid } \\
\text { binding }\end{array}$ & 5 & $3.06 E-03$ \\
\hline & & GO:0030276 clathrin binding & 4 & $1.65 E-02$ \\
\hline & & GO:0042802 identical protein binding & 13 & $2.68 E-02$ \\
\hline & \multirow{3}{*}{ Pathway } & hsa04110:cell cycle & 6 & $6.48 E-03$ \\
\hline & & hsa05222:small cell lung cancer & 4 & $4.75 E-02$ \\
\hline & & hsa05215:prostate cancer & 4 & $4.89 E-02$ \\
\hline
\end{tabular}


TABle 1: Continued.

\begin{tabular}{|c|c|c|c|c|}
\hline & Category & Term & Count & $P$ value \\
\hline \multirow{15}{*}{ Down } & \multirow{4}{*}{$\mathrm{BP}$} & GO:0007267 cell-cell signaling & $1.60 E+01$ & $8.96 E-05$ \\
\hline & & GO:0007155 cell adhesion & $1.90 E+01$ & $9.76 E-04$ \\
\hline & & GO:0070370 cellular heat acclimation & $3.00 E+00$ & $2.07 E-03$ \\
\hline & & GO:0005576 extracellular region & 65 & $6.66 E-11$ \\
\hline & \multirow[t]{2}{*}{$\mathrm{CC}$} & $\begin{array}{c}\text { GO:0005887 integral component of plasma } \\
\text { membrane }\end{array}$ & 51 & $8.76 E-07$ \\
\hline & & GO:0016324 apical plasma membrane & 19 & $6.89 E-06$ \\
\hline & \multirow{3}{*}{ MF } & GO:0005319 lipid transporter activity & 4 & $3.08 E-03$ \\
\hline & & GO:0008083 growth factor activity & 10 & $3.32 E-03$ \\
\hline & & GO:0046982 protein heterodimerization activity & 19 & $3.90 E-03$ \\
\hline & \multirow{6}{*}{ Pathway } & hsa05322:systemic lupus erythematosus & 9 & $5.29 E-03$ \\
\hline & & hsa02010:ABC transporters & 5 & $1.12 E-02$ \\
\hline & & hsa05134:legionellosis & 5 & $2.25 E-02$ \\
\hline & & hsa04080:neuroactive ligand-receptor interaction & 12 & $2.27 E-02$ \\
\hline & & hsa05034:alcoholism & 9 & $2.53 E-02$ \\
\hline & & hsa04975:fat digestion and absorption & 4 & $4.25 E-02$ \\
\hline
\end{tabular}

Up, upregulated genes; down, downregulated genes; BP, biological process; CC, cellar component; MF, molecular function.

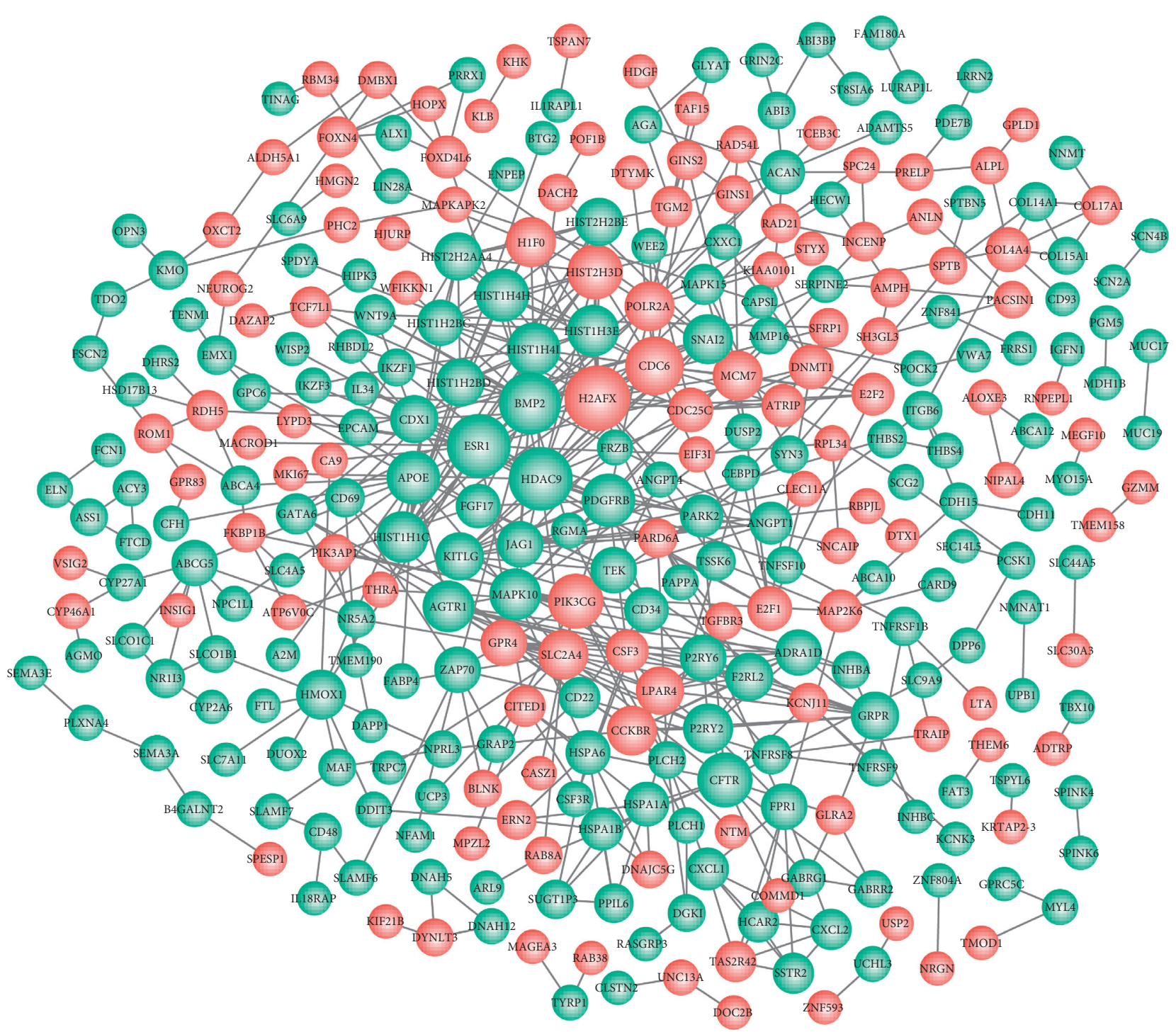

FIGURE 4: Protein-protein interaction (PPI) network construction for differentially expressed genes. A total of 317 nodes and 547 edges were identified. Red, upregulated genes; green, downregulated genes. 
TABle 2: The hub nodes with highest degrees in PPI network.

\begin{tabular}{lc}
\hline Degree & Gene \\
\hline 19 & H2AFX \\
18 & HDAC9, ESR1 \\
17 & BMP2 \\
15 & CDC6 \\
13 & HIST2H3D, CFTR, PIK3CG \\
12 & HIST1H4H, PDGFRB,APOE, HIST1H4I \\
11 & AGTR1, MAPK10, HIST1H3E, HIST1H1C \\
10 & MCM7, HMOX1, SLC2A4, POLR2A, \\
& CCKBR,SNAI2, P2RY2, H1F0 \\
\hline
\end{tabular}

insulin-resistant HepG2 cell model was successfully infected by recombinant adenovirus, suggesting that APOAVoverexpressed HepG2 cell model was built successfully.

5.2. DEGs Screening and Functional Analysis. Microarray technology has been a suitable method for investigating the global gene expression in human disease, which shows an altered gene expression profiling in disease group compared with normal controls [30, 31]. Moreover, there have been studies that used microarray data for investigating gene expression levels about insulin resistance induced by mitochondrial dysfunction, reduced mitochondrial density, and increased IRS-1 serine phosphorylation [32, 33]. Besides, the DAVID gene functional classification tool can be used to condense functionally related gene list to a significant GO or pathway terms with an enrichment score. PPI network was critical to screen the significant gene nodes and understand the functions of predicted genes. Based on microarray experiments, 313 overexpressed genes and 563 low-expressed genes were identified in APOAV overexpressed cells. Hierarchical clustering analysis revealed that the B-M samples and A-Con group were clearly separated based on the differential gene expression profiles, suggesting that the differential genes identified in our paper were significant.

Furthermore, the function analysis showed that the downregulated genes were closely related with fat digestion and absorption pathway. T2D and obesity have been reported to be the metabolic diseases that are characterized by impaired insulin action and insulin resistance induced by low-grade inflammatory [34]. Recent evidence shows that nutritional fatty acids induce inflammatory response in adipocytes and macrophages [35]. Lipopolysaccharides (LPS) are another key inducer for inflammatory response during insulin resistance, which has a coreceptor for fatty acids. The plasma LPS level and LPS absorption are significantly increased during fat digestion [36]. In our study, the pathway of fat digestion and absorption were significantly related with downregulated genes in HepG2 cells induced by APOAV overexpression. Therefore, APOAV overexpression may inhibit the fat digestion and absorption triggered inflammation, and then declined the risk for insulin resistance development.

5.3. PPI Network and Key Genes. The PPI network is critical for understanding the functional modules for genes of interest. In this paper, two functional modules were screened out in the PPI network. Specially, genes of AGTR1 and $P 2 R Y 2$ in module 1 were found to be significantly enriched in G-protein-related signaling functions such as phospholipase C-activating G-protein-coupled receptor signaling pathway (GO:0007200), G-protein-coupled purinergic nucleotide receptor activity (GO:0045028), and G-proteincoupled receptor activity (GO:0004930). The G-protein signaling plays a key role in cell proliferation, cell wall changes, and transcriptional regulation. Besides, more than $50 \%$ drugs are developed by targeting G-protein-coupled receptors (GPCRs), revealing the critical role of G-protein signaling in various human diseases [37]. Moreover, a previous study has revealed that the insulin action is impaired by the deficiency of G-protein subunit Gia2 [38]. Therefore, the alteration in G-protein signaling may contribute to the progression of insulin resistance. However, the role of G-protein function has been not elucidated clearly.

AGTR1 (degree $=11)$ and P2RY2 (degree $=10)$ were found to be significant nodes in PPI network. P2RY2 is one of $\mathrm{P} 2 \mathrm{Y}$ genes and its protein production (purinergic receptor P2Y, G-protein coupled 2) is a member of GPCRs. Based on the description above, the differential expression of $P 2 R Y 2$ in liver cells may contribute to the insulin resistance. AGTR1 is the angiotensin II receptor related gene. The polymorphism of AGTR1 is proposed to affect the insulin resistance by altering the response to angiotensin II signaling [39]. As we all know, hypertension cases are insulin-resistant; AGTR1 polymorphism is also found to be associated with early inflammatory and metabolic changes in prehypertensive cases [40]. Besides, it is reported that the receptor of AGTR1 is activated on liver cells in initiation of insulin resistance, which further enlarges the activation of hepatocellular NF- $\kappa \mathrm{B}$ signaling [41] and NF- $\kappa \mathrm{B}$ signaling is responsible for negative crosstalk with insulin. Although there is no evidence for the relationship between AGTR1 and G-protein signaling, the alteration in AGTR1 expression significantly related with insulin resistance.

It is known that glucose transporters (Gluts) play an important role in glucose regulation [42, 43]. As an insulinregulated Glut, Glut4 mediates the glucose uptake in skeletal muscle cells. An increasing number of researches show that intracellular compartmentalization of GLUT4 is altered in models of insulin resistance [44, 45]. In this paper, cell verification experiments showed that after transfected with the APOAV overexpression vector, the expression of Glut4 in AML12 cell was significantly elevated, which indicated that the overexpression of $A P O A V$ could release the insulin resistance of AML12 cells. Moreover, APOAV overexpression could promote the absorption of glucose in AML12 cells. Taken together, all these evidence validated the role of APOAV expression in altering insulin resistance.

Furthermore, TNFRSF1B was a documented marker gene associated with T2D and was downregulated in liver cells induced by APOAV overexpression. TNF receptor 2 (TNFR2) encoded by TNFRSF1B plays a key role in insulin resistance-related metabolic disorders by mediating the metabolic effect of TNF a. The variable genomic TNFRSF1B 


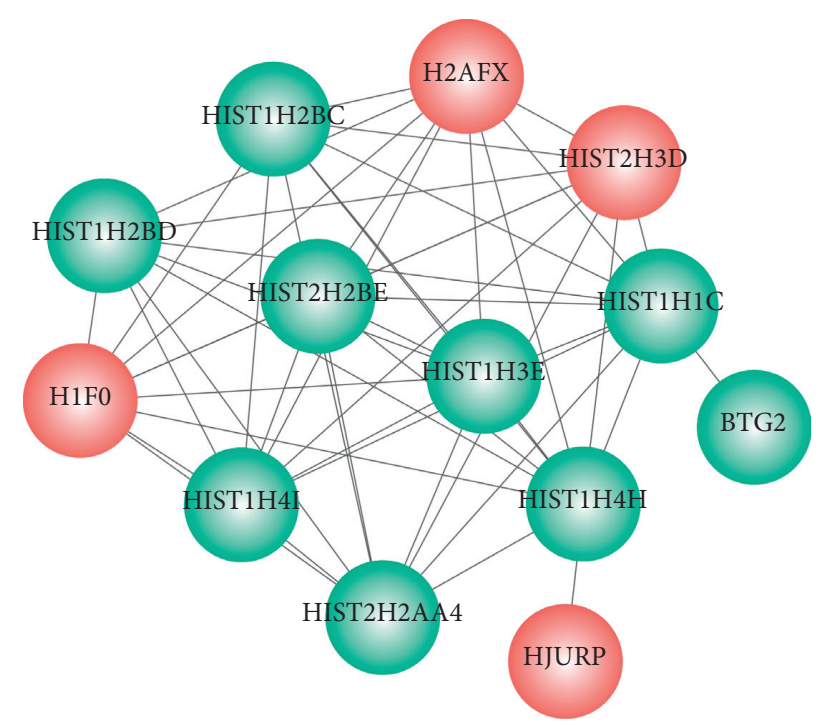

(a)

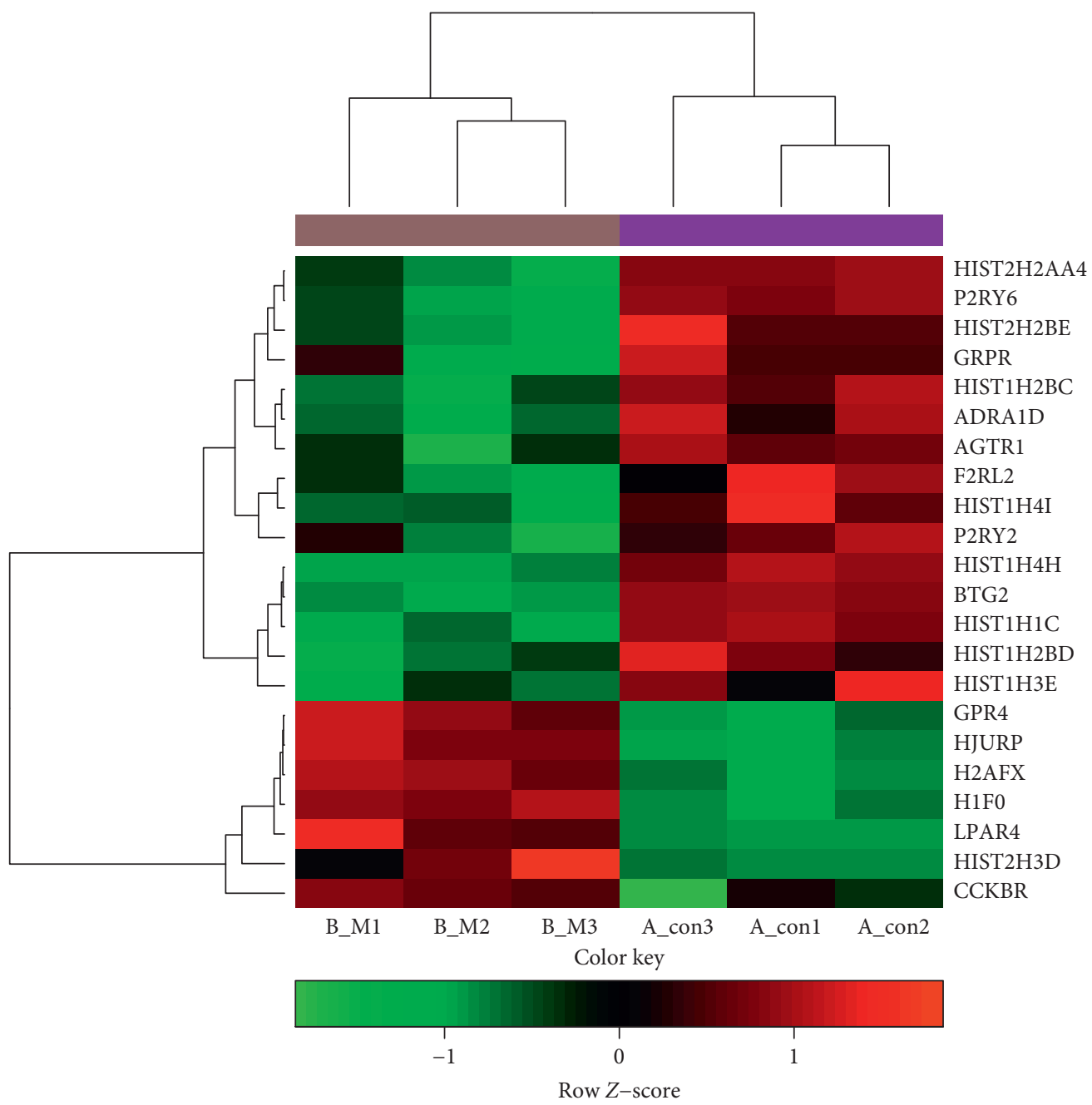

(b)

Figure 5: (a) Functional modules screened in PPI network. Red, upregulated genes; green, downregulated genes; PPI, protein-protein interaction. (b) Hierarchical clustering analysis for module genes. The samples of insulin-resistant HepG2 cells with APOAV overexpression (B-M) and negative controls (A-con) were distinguished clearly by the expression profiles of differentially expressed genes. 
TABLE 3: List of degrees for modular genes in PPI network.

\begin{tabular}{|c|c|c|c|}
\hline \multicolumn{2}{|c|}{ Module 1} & \multicolumn{2}{|c|}{ Module 2} \\
\hline Node & Degree & Node & Degree \\
\hline AGTR1 & 11 & H2AFX & 19 \\
\hline P2RY2 & 10 & HIST2H3D & 13 \\
\hline CCKBR & 10 & HIST1H4I & 12 \\
\hline LPAR4 & 9 & HIST1H4H & 12 \\
\hline F2RL2 & 9 & HIST1H1C & 11 \\
\hline GRPR & 9 & HIST1H3E & 11 \\
\hline GPR4 & 8 & $\mathrm{H} 1 \mathrm{~F} 0$ & 10 \\
\hline ADRA1D & 8 & HIST2H2BE & 9 \\
\hline P2RY6 & 8 & HIST2H2AA4 & 9 \\
\hline- & - & HIST1H2BD & 9 \\
\hline - & - & HIST1H2BC & 8 \\
\hline - & - & HJURP & 1 \\
\hline - & - & BTG2 & 1 \\
\hline
\end{tabular}

PPI: protein-protein interaction.

TABLE 4: Altered GO function and pathways for genes in significant modules.

\begin{tabular}{|c|c|c|c|c|}
\hline & Category & Term & Count & $P$ value \\
\hline \multirow{11}{*}{ Module 1} & \multirow{3}{*}{$\mathrm{BP}$} & $\begin{array}{c}\text { GO:0007200 phospholipase C-activating G-protein } \\
\text { coupled receptor signaling pathway }\end{array}$ & 5 & $7.25 E-09$ \\
\hline & & $\begin{array}{c}\text { GO:0007204 positive regulation of cytosolic calcium } \\
\text { ion concentration }\end{array}$ & 4 & $2.29 E-05$ \\
\hline & & $\begin{array}{c}\text { GO:0007186 G-protein coupled receptor signaling } \\
\text { pathway }\end{array}$ & 5 & $4.98 E-04$ \\
\hline & \multirow{3}{*}{$\mathrm{CC}$} & $\begin{array}{c}\text { GO:0005887 integral component of plasma } \\
\text { membrane }\end{array}$ & 9 & $8.82 E-10$ \\
\hline & & GO:0005886 plasma membrane & 9 & $5.52 E-06$ \\
\hline & & GO:0016324 apical plasma membrane & 3 & $6.78 E-03$ \\
\hline & \multirow{3}{*}{ MF } & $\begin{array}{c}\text { GO:0045028 G-protein coupled purinergic } \\
\text { nucleotide receptor activity }\end{array}$ & 2 & $6.29 E-03$ \\
\hline & & $\begin{array}{c}\text { GO:0004435 phosphatidylinositol phospholipase C } \\
\text { activity }\end{array}$ & 2 & $1.35 E-02$ \\
\hline & & GO:0004930 G-protein coupled receptor activity & 3 & $3.65 E-02$ \\
\hline & \multirow{2}{*}{ Pathway } & hsa04080:neuroactive ligand-receptor interaction & 8 & $1.57 E-10$ \\
\hline & & hsa04020:calcium signaling pathway & 4 & $5.22 E-04$ \\
\hline \multirow{12}{*}{ Module 2} & \multirow{3}{*}{$\mathrm{BP}$} & GO:0006334 nucleosome assembly & 11 & $6.08 E-20$ \\
\hline & & GO:0006325 chromatin organization & 6 & $4.58 E-07$ \\
\hline & & GO:0032776 DNA methylation on cytosine & 4 & $1.76 E-06$ \\
\hline & \multirow{3}{*}{$\mathrm{CC}$} & GO:0000786 nucleosome & 10 & $2.64 E-19$ \\
\hline & & GO:0000788 nuclear nucleosome & 4 & $2.69 E-06$ \\
\hline & & GO:0005654 nucleoplasm & 10 & $3.98 E-06$ \\
\hline & \multirow{3}{*}{ MF } & GO:0046982 protein heterodimerization activity & 9 & $1.97 E-10$ \\
\hline & & GO:0042393 histone binding & 6 & $1.26 E-08$ \\
\hline & & GO:0003677 DNA binding & 10 & $5.40 E-08$ \\
\hline & \multirow{3}{*}{ Pathway } & hsa05322:systemic lupus erythematosus & 9 & $1.47 E-13$ \\
\hline & & Hsa05034:alcoholism & 9 & $1.42 E-12$ \\
\hline & & hsa05203:viral carcinogenesis & 5 & $8.52 E-05$ \\
\hline
\end{tabular}

GO, gene ontology; BP, biological process; CC, cellar component; MF, molecular function.

affects the soluble TNFR2 level. The level of soluble TNFR2 has been found to increase in obese patients, which is correlated with insulin resistance. In this paper, TNFRSF1B level was found to be downregulated mediated by APOAV overexpression, suggesting that APOAV overexpression inhibited TNFR2 involved inflammatory response.
In summary, APOAV overexpression may prevent the development and progression of insulin resistance in HepG2 cells by triggering differential gene expression. APOAV may play a key role in mediating insulin resistance by targeting AGTR1 and P2RY2. Our work may provide a promising option for treating insulin-resistant metabolic diseases. 


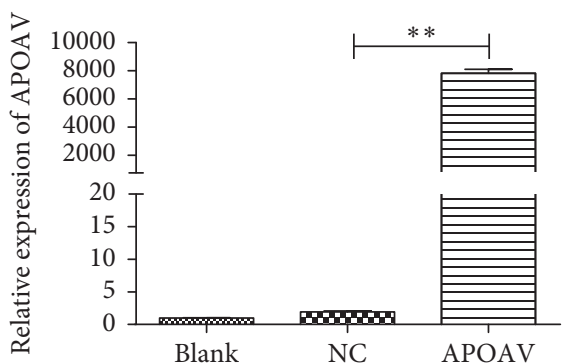

(a)

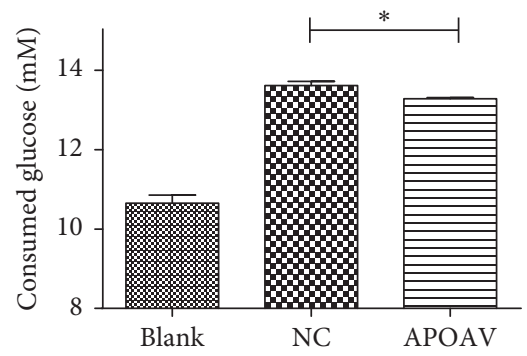

(b)

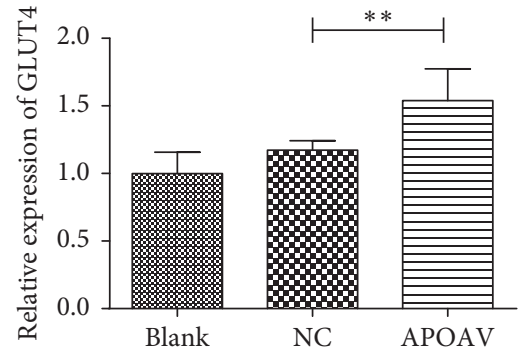

(c)

Figure 6: (a) APOAV expression in the blank group, NC group, and APOAV overexpression group measured by qRT-PCR. (b) Glucose uptake of AML12 cells in the blank group, NC group, and APOAV overexpression group. (c) Glut4 protein levels in the blank group, NC group, and APOAV overexpression group. ${ }^{*} P<0.05,{ }^{* *} P<0.01$.

\section{Data Availability}

The data used to support the findings of this study are included within the article.

\section{Conflicts of Interest}

The authors declare that they have no conflicts of interest.

\section{Acknowledgments}

This work was supported by the National Natural Science Foundation of China (grant no. 81500234).

\section{Supplementary Materials}

Supplementary File 1: the detailed data information of each node in the PPI network (Figure 4). (Supplementary Materials)

\section{References}

[1] A. R. Baker, S. J. Creely, P. G. McTernan, and S. Kumar, "Epicardial and intramyocardial adipose tissue: the enemy within," Immunology, Endocrine \& Metabolic Agents in Medicinal Chemistry (Formerly Current Medicinal ChemistryImmunology, Endocrine and Metabolic Agents), vol. 7, pp. 143-148, 2007.

[2] L. Valenti, R. M. Mendoza, R. Rametta, M. Maggioni, C. Kitajewski, C. J. Shawber et al., "Hepatic notch signaling correlates with insulin resistance and nonalcoholic Fatty liver disease," Diabetes, vol. 62, no. 12, pp. 4052-4062, 2013.

[3] B. C. Martin, J. H. Warram, A. S. Krolewski et al., "Role of glucose and insulin resistance in development of type 2 diabetes mellitus: results of a 25-year follow-up study," The Lancet, vol. 340, no. 8825, pp. 925-929, 1992.

[4] Z. Gao, S. Leng, F. Lu, M. Xie, L. Xu, and K. Wang, "Effect of berberine on expression of hepatocyte nuclear factor- $4 \alpha$ in rats with fructose-induced insulin resistance," Journal of Huazhong University of Science and Technology (Medical Sciences), vol. 28, no. 3, pp. 261-265, 2008.

[5] S. Lehto, T. Rönnemaa, K. Pyörälä, and M. Laakso, "Cardiovascular risk factors clustering with endogenous hyperinsulinaemia predict death from coronary heart disease in patients with type II diabetes," Diabetologia, vol. 43, no. 2, pp. 148-155, 2000.
[6] X.-S. Huang, S.-P. Zhao, M. Hu, L. Bai, Q. Zhang, and W. Zhao, "Decreased apolipoprotein A5 is implicated in insulin resistance-related hypertriglyceridemia in obesity," Atherosclerosis, vol. 210, no. 2, pp. 563-568, 2010.

[7] S. Calandra, C. P. Oliva, P. Tarugi, and S. Bertolini, "APOA5 and triglyceride metabolism, lesson from human APOA5 deficiency," Current Opinion in Lipidology, vol. 17, no. 2, pp. 122-127, 2006.

[8] F. G. Schaap, P. C. N. Rensen, P. J. Voshol et al., "ApoAV reduces plasma triglycerides by inhibiting very low density lipoprotein-triglyceride (VLDL-TG) production and stimulating lipoprotein lipase-mediated VLDL-TG hydrolysis," Journal of Biological Chemistry, vol. 279, no. 27, pp. 2794127947, 2004.

[9] K. W. van Dijk, P. C. Rensen, P. J. Voshol, and L. M. Havekes, "The role and mode of action of apolipoproteins CIII and AV: synergistic actors in triglyceride metabolism?," Current Opinion in Lipidology, vol. 15, no. 3, pp. 239-246, 2004.

[10] M. Nowak, A. Helleboid-Chapman, H. Jakel et al., "Insulinmediated down-regulation of apolipoprotein A5 gene expression through the phosphatidylinositol 3-kinase pathway: role of upstream stimulatory factor," Molecular and Cellular Biology, vol. 25, no. 4, pp. 1537-1548, 2005.

[11] A. Watson, A. Mazumder, M. Stewart, and S. Balasubramanian, "Technology for microarray analysis of gene expression," Current Opinion in Biotechnology, vol. 9, no. 6, pp. 609-614, 1998.

[12] J. Xu, M. Ma, and W. M. Purcell, "Characterisation of some cytotoxic endpoints using rat liver and HepG2 spheroids as in vitro models and their application in hepatotoxicity studies. I. Glucose metabolism and enzyme release as cytotoxic markers," Toxicology and Applied Pharmacology, vol. 189, no. 2, pp. 100-111, 2003.

[13] W. Xie, W. Wang, H. Su, D. Xing, Y. Pan, and L. Du, "Effect of ethanolic extracts of Ananas comosus L. leaves on insulin sensitivity in rats and HepG2," Comparative Biochemistry and Physiology Part C: Toxicology \& Pharmacology, vol. 143, no. 4, pp. 429-435, 2006.

[14] M. D. Robinson, D. J. McCarthy, and G. K. Smyth, "edgeR: a bioconductor package for differential expression analysis of digital gene expression data," Bioinformatics, vol. 26, no. 1, pp. 139-140, 2010.

[15] C. W. Law, Y. Chen, W. Shi, and G. K. Smyth, "Voom: precision weights unlock linear model analysis tools for RNAseq read counts," Genome Biology, vol. 15, no. 2, p. R29, 2014.

[16] M. E. Ritchie, B. Phipson, D. Wu et al., "Limma powers differential expression analyses for RNA-sequencing and 
microarray studies," Nucleic Acids Research, vol. 43, no. 7, p. e47, 2015.

[17] G. K. Smyth, "Limma: linear models for microarray data," in Bioinformatics and Computational Biology Solutions Using $R$ and Bioconductor, pp. 397-420, Springer, Berlin, Germany, 2005.

[18] G. R. Warnes, B. Ben, L. Bonebakker et al., "gplots: various R programming tools for plotting data," $R$ package version, vol. 2, 2009.

[19] Da. W. Huang, B. T. Sherman, and R. A. Lempicki, "Systematic and integrative analysis of large gene lists using DAVID bioinformatics resources," Nature Protocols, vol. 4, pp. 44-57, 2008.

[20] S. Damian, A. Franceschini, M. Kuhn et al., "The STRING database in 2011: functional interaction networks of proteins, globally integrated and scored," Nucleic Acids Research, vol. 2010, Article ID gkq973, 2010.

[21] S. Damian, A. Franceschini, S. Wyder et al., "STRING v10: protein-protein interaction networks, integrated over the tree of life," Nucleic acids research, vol. 2014, Article ID gku1003, 2014.

[22] P. Shannon, A. Markiel, O. Owen et al., "Cytoscape: a software environment for integrated models of biomolecular interaction networks," Genome Research, vol. 13, no. 11, pp. 2498-2504, 2003.

[23] T. Nepusz, H. Yu, and A. Paccanaro, "Detecting overlapping protein complexes in protein-protein interaction networks," Nature Methods, vol. 9, no. 5, pp. 471-472, 2012.

[24] A. P. Davis, C. J. Grondin, K. Lennon-Hopkins et al., "The comparative toxicogenomics database's 10th year anniversary: update 2015," Nucleic Acids Research, vol. 43, no. D1, pp. D914-D920, 2015.

[25] S. L. Fernandez-Valverde, R. J. Taft, and J. S. Mattick, "MicroRNAs in -cell biology, insulin resistance, diabetes and its complications," Diabetes, vol. 60, no. 7, pp. 1825-1831, 2011.

[26] Y. Yan, H. Deng, L. Jian, Y. Su, Y. Li, and G. Mi, “Associations of apolipoprotein A5 with triglyceride, adiponectin and insulin resistance in patients with impaired glucose regulation and type 2 diabetes mellitus," International Journal of Diabetes in Developing Countries, vol. 33, no. 1, pp. 13-17, 2013.

[27] N. Kumashiro and D. M. Erion, D. Zhang et al., Cellular mechanism of insulin resistance in nonalcoholic fatty liver disease," Proceedings of the National Academy of Sciences, vol. 108, no. 39, pp. 16381-16385, 2011.

[28] J. Styskal, H. Van Remmen, A. Richardson, and A. B. Salmon, "Oxidative stress and diabetes: what can we learn about insulin resistance from antioxidant mutant mouse models?," Free Radical Biology and Medicine, vol. 52, no. 1, pp. 46-58, 2012.

[29] M. Takao, "Cell culture carrier and cell culture vessel," Philosophical Transactions of the Royal Society B: Biological Sciences, vol. 370, 2014.

[30] S. Szmit, M. Jank, H. Maciejewski et al., "Gene expression profiling in peripheral blood nuclear cells in patients with refractory ischaemic end-stage heart failure," Journal of Applied Genetics, vol. 51, no. 3, pp. 353-368, 2010.

[31] A. S. Barth, A. Kumordzie, C. Frangakis, K. B. Margulies, T. P. Cappola, and G. F. Tomaselli, "Reciprocal transcriptional regulation of metabolic and signaling pathways correlates with disease severity in heart failure," Circulation: Cardiovascular Genetics, vol. 4, no. 5, pp. 475-483, 2011.

[32] K. F. Petersen, B. Douglas, S. Dufour et al., "Mitochondrial dysfunction in the elderly: possible role in insulin resistance," Science, vol. 300, no. 5622, pp. 1140-1142, 2003.
[33] K. Morino, K. F. Petersen, S. Dufour et al., "Reduced mitochondrial density and increased IRS-1 serine phosphorylation in muscle of insulin-resistant offspring of type 2 diabetic parents," Journal of Clinical Investigation, vol. 115, no. 12, pp. 3587-3593, 2005.

[34] K. E. Wellen and G. S. Hotamisligil, "Inflammation, stress, and diabetes," Journal of Clinical Investigation, vol. 115, no. 5, pp. 1111-1119, 2005.

[35] H. Shi, M. V. Kokoeva, K. Inouye, I. Tzameli, H. Yin, and J. S. Flier, "TLR4 links innate immunity and fatty acid-induced insulin resistance," Journal of Clinical Investigation, vol. 116, no. 11, pp. 3015-3025, 2006.

[36] D. Black, P. Tso, W. Stuart, and S. M. Sabesin, "Intestinal lipoproteins in the rat with $\mathrm{D}-(+)$-galactosamine hepatitis," Journal of Lipid Research, vol. 24, pp. 977-992, 1983.

[37] K. L. Pierce, R. T. Premont, and R. J. Lefkowitz, "Seventransmembrane receptors," Nature Reviews Molecular Cell Biology, vol. 3, no. 9, pp. 639-650, 2002.

[38] K. F. Petersen, S. Dufour, D. Befroy, R. Garcia, and G. I. Shulman, "Impaired mitochondrial activity in the insulin-resistant offspring of patients with type 2 diabetes," New England Journal of Medicine, vol. 350, no. 7, pp. 664-671, 2004.

[39] H. Akasaka, T. Katsuya, S. Saitoh et al., "Effects of angiotensin II type 1 receptor gene polymorphisms on insulin resistance in a Japanese general population: the Tanno-Sobetsu study," Hypertension Research, vol. 29, no. 12, pp. 961-967, 2006

[40] G. M. Reaven, "Role of insulin resistance in human disease," Diabetes, vol. 37, no. 12, pp. 1595-1607, 1988.

[41] M. Van Beek, K. I. Oravecz-Wilson, P. C. Delekta et al., "Bcl10 links saturated fat overnutrition with hepatocellular NF- $\kappa \mathrm{B}$ activation and insulin resistance," Cell Reports, vol. 1, pp. 444-452, 2012.

[42] V. Castrejón, R. Carbó, and M. Martínez, "Mecanismos moleculares que intervienen en el transporte de la glucosa," Reb Revista de Educación Bioquímica, vol. 26, 2007.

[43] A. R. Manolescu, K. Witkowska, A. Kinnaird, T. Cessford, and C. Cheeseman, "Facilitated hexose transporters: new perspectives on form and function," Physiology, vol. 22, no. 4, pp. 234-240, 2007.

[44] K. Dawson, A. Aviles-Hernandez, S. W. Cushman, and D. Malide, "Insulin-regulated trafficking of dual-labeled glucose transporter 4 in primary rat adipose cells," Biochemical and Biophysical Research Communications, vol. 287, no. 2, pp. 445-454, 2001.

[45] A. C. Coster, R. Govers, and D. E. James, "Insulin stimulates the entry of GLUT4 into the endosomal recycling pathway by a quantal mechanism," Traffic, vol. 5, pp. 763-771, 2010. 